\title{
Tipologia de Lesch em alcoolistas no Brasil
}

\author{
Lesch's Typology in Brazilian alcoholic patients \\ Maria da Penha Zago-Gomes', Ester Miyuki Nakamura-Palacios²
}

\section{RESUMO}

Objetivos: Alcoolismo é uma doença heterogênea, com apresentações clínicas, resultados terapêuticos e recaídas variáveis, indicando vulnerabilidades biológicas diferentes. A Tipologia de Lesch distingue quatro categorias de alcoolismo: Tipo I - graves sintomas de abstinência; Tipo II - álcool como solução para conflitos; Tipo III - álcool para "tratamento" de desordens psiquiátricas; Tipo IV - alterações neurológicas antes do uso de álcool. Este estudo verificou a aplicabilidade de uma classificação do tipo clínico de alcoolismo pela Tipologia de Lesch em um ambulatório público brasileiro de atendimento especializado de alta demanda. Método: Estudo seccional descritivo, que classificou pacientes do ambulatório de alcoolismo do Hospital Universitário da Universidade Federal do Espírito Santo de acordo com a Tipologia de Lesch. Resultados: A diferenciação pela Tipologia de Lesch foi facilmente conduzida em um serviço ambulatorial público de alta demanda. De 170 pacientes, 21,2\% foram classificados como Tipo I; 29,4\%, Tipo II; 28,8\%, Tipo III e 20,6\%, Tipo IV. Embora os diferentes tipos de alcoolismo tenham diferentes apresentações clínicas, o padrão de ingestão alcoólica, idade da primeira ingestão e tempo de abstinência não diferiram entre os tipos de alcoolismo. Conclusão: A distinção do tipo clínico de alcoolismo de acordo com a Tipologia de Lesch foi considerada aplicável em um ambulatório público brasileiro de grande demanda, sendo os dados encontrados semelhantes aos relatados em estudos realizados em diferentes países. A aplicação dessa classificação poderá definir mudanças nas estratégias de enfrentamento individualizadas do alcoolismo, sendo, entretanto, necessários estudos de seguimento para avaliar os resultados terapêuticos das mesmas.

\begin{abstract}
Objective: Alcohol dependence is a heterogeneous disease with a clear variability in clinical, therapeutic results and relapses, indicating different biological vulnerability. Lesch et al (1988) distinguished four categories of alcohol dependence: Type I - severe symptoms of abstinence; Type II - alcohol as a solution for conflicts; Type III - alcohol as self-medication for psychiatric symptoms; Type IV - history of neurological lesions preceding the development of alcohol dependence. This study examined the applicability of a classification of the clinical type of alcoholism according to
\end{abstract}

Programa de Pós-graduação em Ciências Fisiológicas da Universidade Federal do Espírito Santo

1 Professora Adjunta do Departamento de Clínica Médica da Universidade Federal do Espírito Santo. Doutorado em Ciências Fisiológicas (área de Neuropsicofarmacologia) pela Universidade Federal do Espírito Santo (2008).

Recebido em

$14 / 5 / 2009$

Aprovado em

2 Professora Associada do Departamento de Ciências Fisiológicas da Universidade Federal do Espírito Santo. Doutorado em Psicobiologia pela Universidade Federal de São Paulo, Brasil (1992), Pós-doutorado pela Louisiana State University System, Estados Unidos (1998), Atuação em Neuropsicofarmacologia.

Endereço para correspondência: Maria da Penha Zago-Gomes

Rua Santa Berenice, n0 55, apto. 301, Praia do Ribeiro - 29101-070 - Vila Velha, ES, Brasil

Telefone: (27) 3329-4333

Telefax: (27) 3335-7215

E-mail: penhazago@gmail.com 


\section{Keywords}

Alcoholism, classification, ambulatory care, diagnose.
Lesch's Typology in a high demanding Brazilian public outpatient service for alcohol dependence. Method: Alcoholics from a Brazilian public outpatient service for treatment of alcoholism of the School of Medicine of the Federal University of Espirito Santo were classified into categories according to Lesch's Typology. Results: Differentiation by Lesch's Typology was easily conducted in a high demanding public outpatient service. Of 170 alcoholics, 21.2\% were classified as Type l; 29.4\%, Type II; 28.8\%, Type III; 20.6\%, Type IV. Although different types of alcoholism have different clinical presentations, patients showed similar pattern of alcohol intake, age for the first use of alcohol and withdrawal. Conclusion: Distinguishing clinical types of alcohol dependence according to Lesch's Typology was considered applicable in a high demanding Brazilian public outpatient service, in which the results were similar to those reported in studies realized in other countries. The application of this classification may provide significant changes in strategies for individualized approaches that may be of a great importance in the treatment of alcohol dependence. However, the therapeutic results of these changes will need to be evaluated in follow-up studies.

\section{INTRODUÇÃO}

O diagnóstico de alcoolismo baseia-se universalmente em critérios clínicos definidos pela Classificação Internacional das Doenças em sua décima versão revisada (CID-10)' e pelo Manual Diagnóstico e Estatístico dos Transtornos Mentais em sua quarta versão (DSM-IV)². No atendimento dos pacientes diagnosticados como alcoolistas são observados distintos quadros clínicos. A variabilidade observada na intensidade da síndrome de abstinência alcoólica (SAA), nos resultados terapêuticos com medicações ou com psicoterapia, na prevenção da recaída, e também nos diferentes resultados de estudos genéticos com alcoolistas, mostra que diferentes vulnerabilidades biológicas aos efeitos do álcool podem existir ${ }^{3-7}$. Reconhecer as diferenças e individualizar o tratamento é importante para se obter sucesso terapêutico.

Após o diagnóstico de alcoolismo ser definido, algumas categorias têm sido propostas, tipificando essa doença de acordo com o comportamento e quadro clínico, a fim de definir condutas distintas ${ }^{8-15}$. Lesch et al. (1988) ${ }^{3}$ apresentaram um estudo prospectivo de 18 anos, com 444 alcoolistas crônicos, acompanhados em média por 5,33 anos no qual observaram dados sociais (família, ocupação, cultura, etc.), personalidade (personalidade aditiva típica ou não típica, comportamento passivo) e uso de álcool (potência da adição, tolerância e uso de outras drogas). Os autores propuseram a classificação do alcoolismo em 4 tipos: I, II, III e IV $4,6,16$, com estratégias distintas de tratamento para cada tipo ${ }^{16}$.

O Tipo I apresenta SAA grave, muitas vezes com crises convulsivas de abstinência. Porém, tem boa adaptação social, participa de atividades de lazer, tem boa relação com familiares, não apresenta distúrbios na infância e não tem história de envolvimento em crimes. Começa o uso de álcool de modo social, e desenvolve uso constante, inclusive para aliviar os sintomas de abstinência. Apresenta compul- são: uma dose leva ao uso adicional de álcool, e existe história familiar de alcoolismo. Esses pacientes podem ser classificados como o "modelo de alergia" e a meta do tratamento é a abstinência total 16,17 .

O Tipo II não apresenta comprometimento somático, e é de difícil diagnóstico. Pode ser confundido com o beber controlado, pois apresenta aparentemente um beber apropriado, tem boa atividade social, apesar da menor atividade de lazer, boa parceria com os pais, porém sofre com o domínio materno. Apresenta comportamento passivo e, quando alcoolizado, muda o comportamento. O álcool seria usado como um "tratamento" para as dificuldades enfrentadas no seu desenvolvimento psíquico, empregado como sedativo para a ansiedade, e muitas vezes consumido em conjunto com drogas sedativas. Esses pacientes podem apresentar moderado grau de dependência e poucas complicações clínicas. São classificados como "modelo de ansiedade", sendo a psicoterapia e tratamentos alternativos possíveis ofertas para terapêuticas desse grupo $0^{16,17}$.

O Tipo III apresenta comprometimento psiquiátrico. Bebe sozinho, apresenta vários problemas sociais com o uso do álcool, envolvendo principalmente sua família com agressões (com e sem álcool), crimes relacionados ao álcool, desemprego frequente, situação social ruim. O álcool é consumido como automedicação para as desordens psiquiátricas. Apresenta tendências autodestrutivas, como pensamentos suicidas com ou sem álcool. A história familiar é positiva para doenças psiquiátricas. Apresenta períodos sem beber ou com uso de álcool mínimo, pois tende a beber quando a doença psiquiátrica está ativa. Frequentemente apresenta agressividade quando está intoxicado, ou mesmo sóbrio. Esses pacientes podem ser classificados como "modelo de depressão". Quando classificados como Tipo III, o tratamento deve se basear na comorbidade psiquiátrica diagnosticada ${ }^{16,17}$.

Os indivíduos classificados como Tipo IV apresentam dano cerebral neonatal ou até a idade de 14 anos, com com- 
plicações somáticas importantes: deterioração cerebral, epilepsia ou polineuropatia. Na infância encontramos história de hipóxia periparto, meningites ou traumas, além de evidências de distúrbios de comportamento (como enurese noturna e gaguejar) e dificuldade social (como dificuldade escolar). Não conseguem ter uso apropriado de álcool, e apresentam grande dificuldade em interrompê-lo. Apresentam graves sinais de intoxicação alcoólica, mesmo com baixas doses de álcool. A evidência de anormalidades psiquiátricas, somáticas e sociais não está limitada aos períodos de consumo de álcool. Histórias de crises convulsivas são relatadas também fora do período de abstinência. Não apresentam atividades de lazer, são geralmente desempregados e envolvem-se com crimes. A relação familiar geralmente apresenta a figura da mãe como dominante. O relacionamento paterno é difícil. Esses pacientes podem ser classificados como "modelo de adaptação" 16,17. Para esse grupo, novas terapêuticas de neurorreabilitação necessitam ser desenvolvidas.

Em 1990, Lesch et al. ${ }^{16}$ publicaram um instrumento como base para a classificação da Tipologia de Lesch de I-IV - sendo esta a primeira tradução para o português -, que recebeu posteriormente a denominação de "árvore decisória" ("decision tree"), instrumento até hoje utilizado para definir a classificação da Tipologia de Lesch (Figura 1).

Doença somática grave antes dos 14 anos

a) Dano perinatal grave

b) Confusão cerebral com sinais neurológicos

c) Outras doenças cerebrais graves

$$
\downarrow
$$

Outras doenças somáticas graves

d) Neuropatia periférica alcoólica grave ou

e) Crises convulsivas sem álcool ou

f) Síndromes cerebrais orgânicas graves (demência) ou

g) Neuropatia periférica moderada e crises convulsivas com o álcool

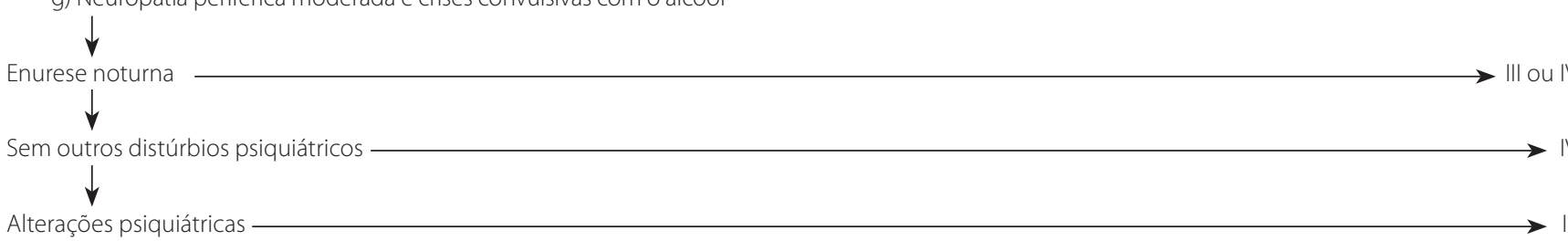

a) Desordens afetivas

b) Oligofrenia

c) Ideias suicidas sem álcool

d) Sintomas depressivos graves nos últimos 6 meses (com alterações graves a moderadas do sono, independentemente do uso de álcool)

Sintomas de abstinência grave

a) Sintomas somáticos moderados ou graves ou

b) Sintomas psíquicos de abstinência moderados ou graves

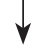

Convulsões no período de abstinência

$\downarrow$

Personalidade passiva

a) Comportamento não agressivo

b) Nenhum ou pouco tempo ocupado

Delinquência com a influência do álcool

Mãe como aliada desculpando-o

Sintomas de comprometimento da família

a) Mãe dominante

b) Melhor relacionamento com o pai do que com a mãe

c) Relacionamento escasso ou ruim com a mãe

S. de abstinência somática e/ou psíquica longa (mais de 6 meses)

Problemas sociais $\longrightarrow$ Checar o diagnóstico do alcoolismo

a) Ofensas sem álcool

b) Multas de trânsito

c) Outras ofensas

$\underset{\downarrow}{\downarrow}$ Alcoolismo crônico? $\longrightarrow$ Teste de metanol

Figura 1. Árvore decisória da classificação da Tipologia de Lesch, adaptada de LESCH et al., 1990. 


\section{OBJETIVO}

O objetivo do presente estudo foi o de verificar a aplicabilidade, em uma população de alcoolistas, atendida em um ambulatório especializado de alta demanda de um serviço público vinculado ao Hospital Universitário da Universidade Federal do Espírito Santo, da árvore decisória de Lesch, para classificar os pacientes dependentes de álcool pela Tipologia de Lesch.

\section{MATERIAIS E MÉTODOS}

Durante o período de dezembro de 2006 a fevereiro de 2007, 170 pacientes consecutivos, atendidos no ambulatório de dependência ao álcool do Hospital Universitário do Centro de Ciências da Saúde da Universidade Federal do Espírito Santo, com diagnóstico de alcoolismo pelo CID-10, foram incluídos no estudo. Através de anamnese dirigida realizada por um único profissional médico clínico, foram coletados dados sociodemográficos (idade, sexo, cor, estado civil, profissão, escolaridade, situação trabalhista e vínculo social), dados sobre o uso de bebidas alcoólicas (idade do início do uso, quantidade de uso médio por dia em gramas, tipo de bebida mais utilizada e tempo de abstinência) e informações necessárias para aplicação da árvore decisória para classificação da Tipologia de Lesch. O estudo foi aprovado pelo Comitê de Ética em Pesquisa e todos os indivíduos assinaram o termo de consentimento.

As análises estatísticas foram feitas por meio do programa estatístico Statistical Package for Social Sciences (SPSS) 8.0 para Windows. Para análise descritiva foi utilizada a média e desvio-padrão da média ou mediana e intervalo interquartil. Para a comparação de dados nominais foi empregado o teste de chi-quadrado. Em todas as análises, foi adotado o valor de $\mathrm{p}<0,05$ para se considerar as diferenças observadas como sendo estatisticamente significantes.

\section{RESULTADOS}

Quando se subdividem os pacientes pela classificação de Lesch, encontramos que, dos 170 pacientes com diagnóstico de alcoolismo, 36 (21,2\%) foram classificados como Lesch Tipo I; 50 (29,4\%) como Lesch Tipo Il; 49 (28,8\%) como Lesch Tipo III e 35 (20,6\%) como Lesch Tipo IV.

Como pode ser observado na Tabela 1, houve uma equivalência de média de idade nos grupos classificados de acordo com a Tipologia de Lesch. A relação masculino/ feminino foi de 9/1 nessa amostra de alcoolistas, e o gênero masculino predominou absoluto no Tipo II de Lesch, enquanto nos pacientes classificados como Tipo III, 28,6\%
Tabela 1. Dados demográficos dos subgrupos de alcoolistas pela classificação de Lesch

\begin{tabular}{|c|c|c|c|c|c|}
\hline & $\begin{array}{c}\text { Lesch } \\
\text { I }\end{array}$ & $\begin{array}{c}\text { Lesch } \\
\text { II }\end{array}$ & $\begin{array}{c}\text { Lesch } \\
\text { III }\end{array}$ & $\begin{array}{c}\text { Lesch } \\
\text { IV }\end{array}$ & $\begin{array}{l}\text { Total } \\
\text { álcool }\end{array}$ \\
\hline $\begin{array}{l}\mathrm{N} \\
(\%)\end{array}$ & $\begin{array}{l}36 \\
(21,2)\end{array}$ & $\begin{array}{l}50 \\
(29,4)\end{array}$ & $\begin{array}{l}49 \\
(28,8)\end{array}$ & $\begin{array}{l}35 \\
(20,6)\end{array}$ & $\begin{array}{l}170 \\
(100)\end{array}$ \\
\hline Sexo:n (\%) & & & $35(71,4)$ & & 148 \\
\hline M & $33(91,7)$ & $50(100)$ & 14 & $30(85,7)$ & $(87,1)$ \\
\hline $\mathrm{F}$ & $3(8,3)$ & - & $(28,6)$ & $5(14,3)$ & $22(12,9)$ \\
\hline \multicolumn{6}{|l|}{$\operatorname{Cor}(\%)$} \\
\hline Branco & 48,6 & 52,0 & 55,1 & 40 & 49,7 \\
\hline Pardo & 42,9 & 42,0 & 38,8 & 40 & 40,8 \\
\hline Preto & 8,6 & 6,0 & 6,1 & 17,1 & 8,9 \\
\hline Indígena & - & - & - & 2,9 & 0,6 \\
\hline \multicolumn{6}{|l|}{ Idade (anos) } \\
\hline Média & 46,4 & 47,9 & 43,8 & 47,1 & 46,4 \\
\hline Desvio-padrão & 11,0 & 9,0 & 9,0 & 9,9 & 9,7 \\
\hline Mediana & 44,5 & 48 & 45 & 47 & 46 \\
\hline Int. Interquartil & $39-57$ & $41-54$ & $36-49$ & $40-53$ & $39-52$ \\
\hline Mín. e Máx. & $20-70$ & $30-74$ & $24-62$ & $33-76$ & $20-76$ \\
\hline \multicolumn{6}{|l|}{ Estado Civil (\%) } \\
\hline União estável & 50,0 & 60,0 & 67,4 & 57,1 & 59,4 \\
\hline Separado & 27,8 & 32,0 & 24,5 & 14,3 & 25,3 \\
\hline Solteiro & 16,7 & 8,0 & 6,1 & 25,7 & 12,9 \\
\hline Viúvo & 5,6 & - & 2,0 & 2,9 & 2,4 \\
\hline \multicolumn{6}{|l|}{ Escolaridade (\%) } \\
\hline Analfabeto & 5,7 & 4,0 & 8,2 & 5,7 & 5,9 \\
\hline Só $1^{\circ}$ ano & - & 6,0 & 2,0 & 25,7 & 7,7 \\
\hline Até $4^{\mathrm{a}}$ série & 45,7 & 42,0 & 34,7 & 37,1 & 39,6 \\
\hline Até $8^{a}$ série & 34,3 & 28,0 & 30,6 & 28,6 & 30,2 \\
\hline Ens. Médio & 14,3 & 14,0 & 24,5 & 2,9 & 14,8 \\
\hline Ens. Superior & - & 6,0 & - & - & 1,8 \\
\hline \multicolumn{6}{|l|}{ Situação trabalhista (\%) } \\
\hline Cart. Assin. & 16,7 & 18,0 & 22,0 & 23,5 & 19,9 \\
\hline S/ Cart. Assin. & 13,9 & 10,0 & 4,9 & 5,9 & 8,7 \\
\hline Autônomo & 47,2 & 26,0 & 24,4 & 20,6 & 29,2 \\
\hline Desempregado & 13,9 & 28,0 & 24,4 & 35,3 & 25,5 \\
\hline Aux. Doença & 2,8 & 4,0 & 14,6 & 5,9 & 6,8 \\
\hline Aposentado & 5,6 & 14,0 & 9,8 & 8,8 & 9,9 \\
\hline \multicolumn{6}{|l|}{ Com quem vive (\%) } \\
\hline Cônjuge & 47,2 & 64,0 & 67,3 & 57,1 & 60,0 \\
\hline Familiares & 47,7 & 20,0 & 16,3 & 28,6 & 25,3 \\
\hline Sozinho & 11,1 & 16,0 & 12,2 & 11,4 & 12,9 \\
\hline Amigos & - & - & 2,0 & 2,9 & 1,2 \\
\hline Outros & - & - & 2,0 & - & 0,6 \\
\hline
\end{tabular}

eram mulheres. Observe-se que, do total da amostra feminina $(n=22)$ na população estudada, $14(63,6 \%)$, a maioria, foram classificadas como Lesch Tipo III, lembrando que esse tipo apresenta um componente depressivo importante, sendo considerado como o "modelo de depressão".

Quanto ao estado civil, curioso observar que mesmo os pacientes classificados como Tipo IV (com história de lesão cerebral prévia ao uso de álcool) apresentam semelhante distribuição no estado civil em relação aos outros tipos, principalmente para a união estável (57,1\%). Com relação à escolaridade e à situação trabalhista, não houve diferenças estatisticamente significantes na comparação entre os grupos e subgrupos (Tabela 1). 
A tabela 2 mostra que não houve diferença estatisticamente significante na idade de início de uso de álcool e na quantidade de álcool ingerida em média por dia, entre os diferentes tipos da classificação de Lesch. O tempo de abstinência apresentou grande variação, porém essa característica não diferiu significativamente entre os subgrupos da Tipologia de Lesch. Quando se analisaram os dados de gravidade da dependência ao álcool, observou-se que, entre os subgrupos classificados de acordo com a Tipologia de Lesch, a maior parte dos pacientes dos Tipos I, III e IV apresentava dependência grave $(97,2 \%, 75,5 \%$ e $80 \%$, respectivamente), sendo que a maioria do Tipo II (56\%) apresentava dependência moderada.

\section{DISCUSSÃO}

Em 1996 Lesch $^{3}$ observou que, ao se analisarem resultados de estudos com novas drogas ou estratégias de tratamento para o alcoolismo, se os pacientes alcoolistas não tiverem sido adequadamente diferenciados entre os subgrupos de manifestações clínicas, estar-se-á avaliando uma população heterogênea, e os resultados de terapias efetivas poderão não ser devidamente identificados.

A distribuição dos alcoolistas classificados pela Tipologia de Lesch em alguns trabalhos publicados está resumida na Tabela 3. Podemos observar algumas diferenças, provavelmente decorrentes do local de origem dos pacientes (se internados ou ambulatoriais) e também as diferentes regiões onde foi realizada a pesquisa, influenciada pela diversidade socioeconômica e cultural. A distribuição dos tipos de alcoolismo encontrados no presente estudo pode ser comparada com os dados de literatura, principalmente aqueles obtidos em população atendida em ambulatório ${ }^{17}$. As diferenças regionais encontradas também poderiam ser devidas a erros na
Tabela 2. Características do uso de bebidas alcoólicas pelos subgrupos de alcoolistas pela classificação de Lesch e controles não alcoolistas

\begin{tabular}{|c|c|c|c|c|c|}
\hline & $\begin{array}{l}\text { Lesch } \\
1\end{array}$ & $\begin{array}{l}\text { Lesch } \\
\text { II }\end{array}$ & $\begin{array}{l}\text { Lesch } \\
\text { III }\end{array}$ & $\begin{array}{l}\text { Lesch } \\
\text { IV }\end{array}$ & $\begin{array}{l}\text { Total } \\
\text { álcool }\end{array}$ \\
\hline N & 36 & 50 & 49 & 35 & 170 \\
\hline \multicolumn{6}{|l|}{ Idade início de uso (anos) } \\
\hline Média & 14,7 & 15,3 & 14,9 & 14,7 & 14,9 \\
\hline Mediana & 14,5 & 15,5 & 15 & 15 & 15 \\
\hline Desvio-padrão & 4,0 & 4,8 & 5,4 & 4,0 & 4,6 \\
\hline Intervalo Interquartil & $12-17,5$ & $12-17,5$ & $10,5-17,5$ & $12-18$ & $12-18$ \\
\hline Mín. e Máx. & $7-25$ & $7-27$ & $6-30$ & $6-21$ & $6-30$ \\
\hline \multicolumn{6}{|l|}{$\begin{array}{l}\text { Quantidade de álcool } \\
\text { (gramas/dia) }\end{array}$} \\
\hline Média & 368,9 & 299,8 & 478,9 & 344,0 & 374,5 \\
\hline Mediana & 360 & 200 & 400 & 240 & 320 \\
\hline Desvio-padrão & 272,5 & 246,9 & 404,6 & 242,8 & 309,8 \\
\hline Intervalo Interquartil & $200-400$ & $120-400$ & $245-475$ & $200-400$ & $190-400$ \\
\hline Mín e Máx & $80-1200$ & $60-1200$ & $80-2400$ & $60-800$ & $60-2400$ \\
\hline \multicolumn{6}{|l|}{ Tempo de abstinência (dias) } \\
\hline Média & 241,3 & 136,3 & 1612,0 & 269,6 & 193,4 \\
\hline Mediana & 56,5 & 35 & 30 & 10 & 36 \\
\hline Desvio-padrão & 457,5 & 312,2 & 395,8 & 570,7 & 429,6 \\
\hline Intervalo Interquartil & $11,5-202,5$ & $6,5-112,5$ & 7-91 & $2-300$ & $5-142,5$ \\
\hline Mín. e Máx. & $0-2160$ & $0-1710$ & $1-2520$ & $0-2880$ & $0-2880$ \\
\hline \multicolumn{6}{|l|}{$\begin{array}{l}\text { Abstinência } \\
\text { categorizada (\%) }\end{array}$} \\
\hline Bebeu no dia & 2,8 & 2,0 & - & 20,0 & 2,4 \\
\hline 1 a 14 dias & 22,2 & 36,0 & 41,7 & 5,7 & 36,7 \\
\hline 15 a 30 dias & 8,3 & 6,0 & 10,4 & 45,7 & 7,1 \\
\hline 31 a 90 dias & 30,6 & 28,0 & 22,9 & 2,9 & 24,3 \\
\hline 3 a 6 meses & 8,3 & 10,0 & 4,2 & 14,3 & 6,5 \\
\hline 6 a 12 meses & 11,1 & 12,0 & 6,3 & 2,9 & 9,5 \\
\hline Mais de 1 ano & 16,7 & 6,0 & 14,6 & 8,6 & 13,6 \\
\hline \multicolumn{6}{|l|}{ Bebida mais utilizada (\%) } \\
\hline Aguardente & 69,4 & 56,0 & 49,0 & 71,4 & 60,0 \\
\hline Cerveja & 2,8 & 14,0 & 4,1 & 8,6 & 7,6 \\
\hline Outros & - & 6,0 & 2,0 & 2,9 & 3,6 \\
\hline Qualquer um & 19,4 & 4,0 & 26,5 & 5,7 & 14,1 \\
\hline Dois tipos & 8,3 & 18,0 & 18,5 & 11,4 & 14,7 \\
\hline \multicolumn{6}{|l|}{ Gravidade do alcoolismo (\%) } \\
\hline Leve & - & 6,0 & - & 2,9 & 2,4 \\
\hline Moderado & 2,8 & 56,0 & 24,5 & 17,1 & 27,6 \\
\hline Grave & 97,2 & 38,0 & 75,5 & 80,0 & 70,0 \\
\hline
\end{tabular}

Tabela 3. Distribuição dos subgrupos de alcoolistas pela classificação de Lesch em alguns dos trabalhos publicados, observando o país onde foi realizada a pesquisa e a origem dos pacientes incluídos (ambulatoriais ou internados)

\begin{tabular}{|c|c|c|c|c|c|c|c|}
\hline Autores/Ano & País & Origem & N & Lesch I (\%) & Lesch II (\%) & Lesh III (\%) & Lesch IV (\%) \\
\hline Lesch e Walter, $1996^{4}$ & Áustria & ID & 260 & 37,3 & 23,5 & 22,3 & 18,1 \\
\hline Wober et al., $1998^{17}$ & Áustria & ID & 82 & 20,7 & 25,6 & 31,7 & 21,9 \\
\hline Chick et al., $2000^{19}$ & Áustria & ID & 149 & 25,0 & 18,0 & 15,0 & 42,0 \\
\hline Sperling et al., $2000^{18}$ & Alemanha & ID e A & 250 & 25,6 & 35,6 & 18,4 & 20,4 \\
\hline Belich et al. $2004^{20}$ & Alemanha & ID & 144 & 20,1 & 50,3 & 19,4 & 10,3 \\
\hline Hillermacher et al., $2006^{21}$ & Alemanha & ID & 192 & 19,3 & 48,9 & 19,8 & 12,0 \\
\hline Walter et al., $2006^{6}$ & Áustria e Bélgica & NR & 159 & 11,3 & 55,3 & 22,0 & 11,3 \\
\hline Bonsch et al., $2006^{22}$ & Alemanha & IC & 134 & 19,4 & 48,5 & 43,3 & 13,5 \\
\hline Samochowiec et al., $2006^{23}$ & Polônia & NR & 100 & 60,0 & 20,0 & 17,0 & 03,0 \\
\hline Reulbach et al., $2007^{24}$ & Alemanha & HP & 48 & 16,7 & 33,3 & 37,5 & 12,5 \\
\hline Samochowiec et al., $2007^{25}$ & Polônia & ID & 122 & 47,5 & 29,5 & 9,0 & 14,0 \\
\hline Biermann et al., $2009^{26}$ & Alemanha & $A$ & 76 & 25,0 & 47,4 & 17,1 & 10,5 \\
\hline Pombo e Lesch, $2009^{27}$ & Portugal & ID & 318 & 19,7 & 35,0 & 37,3 & 24,6 \\
\hline Zago-Gomes e Nakamura-Palacios & Brasil & $A$ & 170 & 21,2 & 29,4 & 28,8 & 20,6 \\
\hline
\end{tabular}

ID = Internação para tratamento de dependência; $A=$ Tratamento de dependência ambulatorial; $H P=$ Hospital psiquiátrico; $\mid \mathrm{C}=$ Internação clínica; NR= não relatado. 
aplicação da árvore decisória de Lesch, embora, para médico clínico ou psiquiátrico treinado, o fluxograma classificatório é de fácil execução. De fato, no atual trabalho não houve nenhuma dificuldade de sua realização em um ambulatório de atendimento público de grande demanda. Lesch e Walter $(1996)^{4}$ ressaltaram a importância da correta classificação dos diferentes subtipos de alcoolismo. Esses autores indicam que a correta observância da árvore decisória de classificação necessita ser seguida para homogeneização dos resultados.

O tratamento do alcoolismo ainda é um grande desafio para os profissionais que trabalham com dependência. Poucos pacientes beneficiam-se apenas de orientações seguras de um profissional sobre a doença. Ao contrário, a maioria necessita de abordagens terapêuticas diferentes. A compreensão das diferenças individuais pode permitir melhores resultados no tratamento da dependência ao álcool.

A classificação da Tipologia de Lesch é passível de ser aplicada por médico treinado, sendo identificada normalmente em uma anamnese bem feita, e poderia ser adotada na rotina das unidades de atendimento de dependência ao álcool para nortear a terapêutica. Entretanto, estudos de seguimento serão necessários para avaliar se mudanças nas estratégias individuais de enfrentamento do alcoolismo resultarão em melhor eficácia no resultado final do tratamento dessa importante doença.

\section{REFERÊNCIAS}

1. Organização Mundial da Saúde (World Health Organization). Classificação de transtornos mentais e de comportamento da CID-10. Descrições clínicas e diretrizes diagnósticas. Tradução da edição brasileira: Dorgival Caetano. Porto Alegre: Artes Médicas; 1993.

2. Associação Americana de Psiquiatria (American Psychiatric Association). Manual diagnóstico e estatístico de transtornos mentais. $4^{\mathrm{a}}$ ed. (DSM-IV). Tradução da edição brasileira: Jorge, MR. Porto Alegre: Artmed; 2002.

3. Lesch OM, Dietzel M, Musalek M, Walter H, Zeiler K. The course of alcoholism. Long-term prognosis in different types. Forensic Sci Int. 1988 Jan;36(1-2):121-38.

4. Lesch OM, Walter H. Subtypes of alcoholism and their role in therapy. Alcohol Alcohol Suppl. 1996 Mar; ;:63-7.

5. Cardoso JM, Barbosa A, Ismail F, Pombo S. NETER alcoholic typology (NAT). Alcohol Alcohol. 2006 Mar-Apr;41(2):133-9.

6. Walter H, Ramskogler K, Semler B, Lesch OM, Platz W. Dopamine and alcohol relapse: D1 and $\mathrm{D} 2$ antagonists increase relapse rates in animal studies and in clinica trial. J Biomed Sci. 2001 Jan-Feb;8(1):83-8.

7. Kiefer F, Barocka A. Secondary depression in weaned alcoholics: implications of Lesch's typology of chronic alcoholism. Alcohol Alcohol. 1999 Nov-Dec;34(6):916-7.
8. Jellinek EM. The disease concept of alcoholism. New Haven: College and University Press; 1960.

9. Cloninger CR, Bohman M, Sigvardsson S. Inheritance of alcohol abuse. Cross-fostering analysis of adopted men. Arch Gen Psychiatry.1981;38(8):861-8.

10. Schuckit MA. The clinical implications of primary diagnostic groups among alcoholics. Arch Gen Psychiatry.1985;42(11):1043-9.

11. Morey LC, Skinner HA. Empirically derived classifications of alcohol-related problems. Recent Dev Alcohol. 1986;:145-68.

12. Wober C, Wober-Bingol C, Karwautz A, Nimmerrichter A, Walter H, Deecke L. Ataxia of stance in different types of alcohol dependence - a posturographic study. Alcohol Alcohol. 1998;33(4):393-402.

13. Zucker RA. The four alcoholisms: a developmental account of the etiologic process. Nebr Symp Motiv. 1986;34:27-8.

14. Babor Tf, Dolinsky Zs, Meyer Re, Hesselbrock M, Hofmann M, Tennen H. Types of alcoholics: concurrent and predictive validity of some common classification schemes. Br J Addict. 1992;87(10):1415-31

15. Hauser J, Rybakowski J. Three clusters of male alcoholics. Drug Alcohol Depend. 1997 Dec 15;48(3):243-50

16. Lesch OM, Kefer J, Lentner S, Mader R, Marx B, Musalek M, et al. Diagnosis of chronic alcoholism-classificatory problems. Psychopathology. 1990;23(2):88-96.

17. Sperling W, Frank H, Martus P, Mader R, Barocka A, Walter H, et al.. The concept of abnormal hemispheric organization in addiction research. Alcohol Alcohol. 2000 JulAug;35(4):394-9.

18. Chick J, Howlett H, Morgan MY, Ritson B. United Kingdom Multicentre Acamprosate Study (UKMAS): a 6-month prospective study of acamprosate versus placebo in preventing relapse after withdrawal from alcohol. Alcohol Alcohol. 2000 Mar-Apr;35(2):176-87.

19. Bleich S, Bayerlein K, Reulbach U, Hillemacher T, Bönsch D, Mugele B, et al. Homocysteine levels in patients classified according to Lesch's typology. Alcohol Alcohol. 2004 NovDec;39(6):493-8.

20. Hillemacher T, Bayerlein K, Wilhelm J, Bönsch, D Poleo D, Sperling W, Kornhuber J, Bleich S. Recurrent detoxifications are associated with craving in patients classified as type 1 according to Lesch's typology. Alcohol Alcohol. 2006;41(1):66-9.

21. Bonsch D, Bayerlein K, Reulbach U, Fiszer R, Hillemacher T, Sperling W, et aç;. Different allele-distribution of mthfr 677 C $\rightarrow$ T Tand mthfr -393 C $\rightarrow$ a in patients classified according to subtypes of Lesch's typology. Alcohol Alcohol. 2006;41(4):364-7.

22. Samochowiec J, Kucharska-Mazur J, Grzywacz A, Jabłoński M, Rommelspacher H, Samochowiec A, et al. Family-based and case-control study of DRD2, DAT, 5HTT, COMT genes polymorphisms in alcohol dependence. J Neurosci Lett. 2006 Dec 13;410(1):1-5.

23. Reulbach U, Biermann T, Bleich S, Hillemacher T, Kornhuber J, Sperling W. Alcoholism and homicide with respect to the classification systems of Lesch and Cloninger. Alcohol Alcohol. 2007 Mar-Apr;42(2):103-7.

24. Samochowiec J, Kucharska-Mazur J, Grzywacz A, Pelka-Wysiecka J, Mak M, Samochowiec A, et al. Genetics of Lesch's typology of alcoholism. Prog Neuropsychopharmacol Biol Psychiatry. 2008 Feb 15;32(2):423-7.

25. Biermann T, Reulbach U, Lenz B, Muschler M, Sperling W, Hillemacher T, et al. Herp mRNA expression in patients classified according to Lesch's typology. Alcohol Alcohol. 2009 Mar;:43(2):91-5.

26. Pombo S, Lesch OM. The alcoholic phenotypes among different multidimensional typologies: similarities and their classification procedures. Alcohol Alcohol. 2009 JanFeb;44(1):46-54. 